\title{
Biometrie - zwischen Pragmatik und Forschung?
}

Seit etwa 20 Jahren wird die Anwendung biometrischer Verfahren zur Unterstützung der Wiedererkennug von Personen in elektronischen Systemen untersucht. Wie für ein neuartiges (und schillerndes) Konzept üblich, sind in diesem Zeitraum zahlreiche Szenarien erprobt und auch wieder verworfen worden. Dabei haben sich wichtige Grundlagen konsolidiert und es sind die ersten Standards als stabile Basis für geschlossene und offene Anwendungen geschaffen worden.

In das Bewusstsein der, Informationsgesellschaft' ist die Biometrie getreten, als nach dem 11.09.2001 ihr vermeintliches Sicherheitspotential von zuständigen Politikern aufgerufen wurde und die Integration von biometrischen Daten (Gesichtsbilder und Fingerabdruckbilder) in neue Identifikationsdokumente mit großem Nachdruck - weltweit - betrieben wird. Mit diesem Prozess ist gleichzeitig ein hoher Erfolgsdruck für ausgewählte biometrische Verfahren aufgebaut worden, der die biometrische Forschungs- und Standardisierungssystematik wesentlich verändert hat.

Dem potenziell großen Spektrum von weiteren biometrischen Merkmalen fehlen nun oft eine adäquate wissenschaftliche Begleitforschung und die Bewertung der Sicherheitsqualitäten der mit innen gestalteten Anwendungen. Ähnliches gilt für den Datenschutz in diesen Lösungen. Ohne Zweifel sind aber die eher pragmatischen Anwendungen der Biometrie Bausteine, die dazu beitragen, zukünftige - vom Markt und den Marktteilnehmern akzeptierte Angebote zu gestalten.

Das aktuelle Heft der DuD widmet sich deshalb nach zwei Jahren erneut dem Thema Biometrie, wobei sowohl Verfahren und Modalitäten diskutiert werden, als auch wie im Beitrag von Matthias Pocs die rechtlichen Rahmenbedingungen für den Betrieb von biometrischen Identifikationssystemen untersucht werden. Die technische und datenschutzbezogene Diskussion ist nicht beschränkt auf die klassischen Verfahren der Fingerbild- und Gesichtserkennung. Florian Dotzler untersucht in seinem Beitrag die datenschutzfreundliche Ausgestaltung von biometrischen Systemen, die das Tippverhalten analysieren und einen textabhängigen Referenzdatensatz hinterlegen.

Ein ebenfalls dynamisches biometrisches Merkmal wird bei der Stimmerkennung analysiert, die im Beitrag von Gereon Tillenburg vorgestellt wird.

Aber auch neue Perspektiven für etablierte Modalitäten werden in diesem Heft behandelt. So stellt Dieter Koller einen Ansatz vor, mit dem ein kontaktlos und 3D-erfasstes Fingerbild durch eine photometrische und geometrische Projektion verarbeitet wird, so dass entstehende Bilder interoperabel mit klassischen Bildern optischer Sensoren verglichen werden können. Auch das wichtige Thema Template Protection wird am Beispiel der Fingerbilderkennung behandelt und gibt einen Einblick in dieses wichtige Forschungsgebiet. Mit dem Beitrag von Martin Drahanský wird über einen ganz neuen Weg in der Forschung berichtet, bei dem die biometrische Erkennung durch eine 3D-Vermessung der Hand erfolgt.

Neben der Forschung spielen die Erfahrungen mit aktuellen Produkten eine wichtige Rolle: Im Anwendungsbeitrag berichtet Uwe Weigmann darüber, wie biometrische Verfahren in die Zugangssicherung zu Sicherheitsbereichen an Flughäfen eingebunden werden und welche Kriterien bei der Auswahl einer geeigneten Technologie zum Einsatz kam.

Christoph Busch, Helmut Reimer 